\title{
Energetic Costs of Cognitive Abilities: Testing the Expensive Tissue Hypothesis
}

\author{
Marek Konarzewski ${ }^{1}$, Anna Goncerzewicz ${ }^{2}$, Ewelina Knapska ${ }^{2}$, Jakub Dzik², and Tomasz \\ Górkiewicz $^{2}$ \\ ${ }^{1}$ University of Bialystok \\ ${ }^{2}$ Nencki Institute of Experimental Biology
}

May 28, 2020

\begin{abstract}
Enlarged brains of homeotherms bring behavioural advantages, but incur high energy expenditures for the animal. The 'Expensive Tissue' (ET) hypothesis links the evolution of the enlarged brain to increased cognitive abilities (CA) that improved foraging performance, social interactions and allowed for reduction in size of the energetically demanding gut. We tested the directionality of the evolutionary trade-off between brain, gut and CA using experimental evolution model consisting of lines of laboratory mice subjected to artificial selection on basal (BMR) or maximum (VO2max) aerobic metabolism - traits that are implicated in evolution of homeothermy and CA. High BMR mice had bigger guts, but not brains. Yet, they performed better in cognitively demanding tasks and had higher neuronal plasticity than their counterparts. The data indicate that evolutionary increase of CA was initially associated with brain plasticity and fuelled by an enlarged gut, which was not traded off for brain size, as the ET posits.
\end{abstract}

\begin{abstract}
Enlarged brains of homeotherms bring behavioural advantages, but incur high energy expenditures for the animal. The 'Expensive Tissue' (ET) hypothesis links the evolution of the enlarged brain to increased cognitive abilities (CA) that improved foraging performance, social interactions and allowed for reduction in size of the energetically demanding gut. We tested the directionality of the evolutionary trade-off between brain, gut and CA using experimental evolution model consisting of lines of laboratory mice subjected to artificial selection on basal (BMR) or maximum $\left(\mathrm{VO}_{2 \max }\right)$ aerobic metabolism - traits that are implicated in evolution of homeothermy and CA. High BMR mice had bigger guts, but not brains. Yet, they performed better in cognitively demanding tasks and had higher neuronal plasticity than their counterparts. The data indicate that evolutionary increase of CA was initially associated with brain plasticity and fuelled by an enlarged gut, which was not traded off for brain size, as the ET posits.
\end{abstract}

Introduction

Many studies suggest that the observed variation in in brain size is ecologically adaptive and maintained by selective trade-offs (e.g., Kotrschal et al. 2015, Pontzer et al . 2016, Sayol et al. 2018). Since increased brain size imposes disproportional metabolic costs, it is likely that those trade-offs involve other metabolically expensive organs. The 'Expensive Tissue' hypothesis (ET) posits that encephalisation was primarily possible thanks to 'financing' metabolic costs of brain maintenance by reducing the size of energetically demanding gut parts (Aiello \& Wheeler 1995). Such reduction was in turn possible by increased cognitive abilities that allowed for more efficient foraging for food of better quality.

The ET scenario is difficult to test because of the lack of palaeontological record, that could be used to analyse the presumed brain-gut trade-off. Evolutionary plausibility of this trade-off can therefore only be 
tested if it is a more general evolutionary principle applicable to extant animals characterized by positive association between enlarged brains and enhanced cognitive abilities. The existence of the brain-gut tradeoff has been questioned in a thorough comparative analysis of brain size and internal organ mass in 100 mammalian species, including 23 primates (Navarrete et al. 2011). The trade-off is also difficult to reconcile with a positive association between brain size and basal metabolic rate, BMR (Isler \& van Schaik, 2006) - a measure of aerobic metabolism reflecting in large part metabolic costs of maintenance of the gut (Konarzewski \& Diamond, 1995). Furthermore, brain size and cognitive abilities are positively correlated with aerobic exercise capacity essential for sustaining such important activities as reproduction or escape from predators (Koteja 2004; Chrząścik et al . 2014; Książek et al. 2009) that must ultimately be fuelled by the gut; these results are also incompatible with the brain-gut trade-off.

To date, studies on the ET hypothesis predominantly used comparative methods (Navarrete et al. 2011). A stronger test of the ET and its associations with cognitive abilities is provided by artificial selection experiments, because they allow for inferences about causal relationships (Garland \& Rose 2009). The most pertinent animal model of this kind was developed by Kotrschal et al ., who demonstrated the brain-gut trade-off in guppies (Poecilia reticulata) artificially selecting for relative brain size (but see Healy \& Rowe 2013). However, life history and physiology of fish is far removed from that of homeotherms (Rose et al. 1993), therefore its relationship with selection on encephalisation in, for example, mammals is questionable. Furthermore, brain size alone does not provide sufficient information to determine cognitive abilities (Healy \& Rowe 2007).

Here, for the first time, we tested the directionality of evolutionary trade-offs between the size and function of the brain and other energetically expensive organs in a mammalian model of experimental evolution. We used line types of laboratory mice subjected to artificial selection on divergent rates of basal (BMR) or maximum aerobic metabolism $\left(\mathrm{VO}_{2 \max }\right.$, Książek et al. 2004; Gębczyński \& Konarzewski 2009)- traits widely accepted as pre-requisites for the evolution of homeothermy and large brain size (Benett \& Ruben, 1979; Lovegrove, 2017).

Materials and Methods

Animals

We used female mice from two concurrent selection experiments carried out at the Faculty of Biology, University of Bialystok. In the first experiment we divergently select mice for high/low body mass-corrected Basal Metabolic Rate (BMR) quantified according to the procedure outlined below. In this experiment we maintain two non-replicated line types: high BMR (H-BMR) and low BMR (L-BMR) line type, whose divergence is sufficiently large to be confidently attributed to the results of selection, rather than to genetic drift (Sadowska et al. 2017). Here we used females of generation F52 and F53.

We also used female mice of generation F37 and F38 from the second selection experiment, in which we established eight genetically isolated Swiss-Webster laboratory mice lines. In four of the lines, forming the Peak Metabolic Rate (PMR) line type, mice were selected for $\mathrm{VO}_{2 \max }$ quantified as the highest body-masscorrected oxygen consumption averaged over $2 \mathrm{~min}$ of a $5 \mathrm{~min}$ swim in a $25^{\circ} \mathrm{C}$ water. The other four lines form the randomly bred (RB), control line type (Gębczyński \& Konarzewski 2009). Throughout the experiment animals were fed a standard diet $(12.8 \mathrm{~kJ}$ g-1 metabolisable energy, $17.0 \mathrm{~kJ}$ g-1 caloric value manufactured by Labofeed, Kcynia, Poland; for detailed composition see (Sadowska et al. 2017).

All procedures were approved by the by the Local Ethical Committee on Testing Animals, (permit no. 42/2011, 11/2013, 21/2013, 194/2016).

Measurements of Basal Metabolic Rate

We simultaneously used two positive pressure, open circuit respirometry systems fitted to two Sable Systems FC-1B oxygen analysers. In each system the outside atmospheric air was pushed through a column of Drierite to remove water vapour and then forced through a copper coil submerged along with metabolic chambers (each $350 \mathrm{cc}$ in volume) in a water bath stabilized at $32{ }^{\circ} \mathrm{C}$ (a temperature that is within our 
animals' thermoneutral zone) to equalize and control the temperature. The air stream was then divided to three independent streams, each fed at $400 \mathrm{~mL} \mathrm{~min}^{-1}$ to a separate mass flow controller (Sierra Instruments, Monterey, CA or ERG1000, Warsaw, Poland) and forced through individual metabolic chambers, and further through a computer-controlled channel multiplexer (Sable Systems, Las Vegas, NV). Air was thereafter scrubbed of $\mathrm{CO}_{2}$ Carboabsorb AS, BDH Laboratory Supplies), dried one more time (Drierite), subsampled at the rate of $75 \mathrm{~mL} \mathrm{~min}{ }^{-1}$, and fed to an oxygen analyzer. BMR was calculated Withers' equation no. 4a (22) and defined as the lowest stable reading that did not vary by more than $0.01 \%$ of oxygen concentration for at least $4 \mathrm{~min}$.

Measurements of cognitive abilities

Following BMR measurements the mice were tested in an automated learning apparatus, an IntelliCage system, from TSE Systems, Germany (Galsworthyet al . 2005; Knapska et al . 2006). The IntelliCage consists of a large standard cage $20.5 \mathrm{~cm}$ high, $40 \mathrm{~cm} \times 58 \mathrm{~cm}$ at the top and $55 \mathrm{~cm} \times 37.5 \mathrm{~cm}$ at the base. The cage is equipped with four operant learning chambers fitted into the corners of the housing cage. Access into the chamber is only possible through a tube with a built-in transponder codes reader (antenna) that restricts access to the learning chamber to only a single mouse at a time. Each corner, equipped with proximity sensor, contains two openings permitting access to drinking bottles. An automatically operated door controls access to liquid. Poking a nose into the openings (nosepoke response) activates an infra-red beam-break response detector. Each visit to the operant chamber, as well as each nosepoke and the amount of water consumed (number and duration of licks) is recorded for each individual animal. The cage control unit permits the access to particular bottles according to schedules individually pre-programmed for each mouse. The cage is equipped with a sleeping shelter in the centre, with a feeder placed on its top providing food ad libitum . Except for the technical breaks and cage exchange (once a week), the mice were not disturbed.

A week before the experiment the mice were sedated and injected with a glass-covered microtransponder (11.5mm length, $2.2 \mathrm{~mm}$ diameter; DataMars) with a unique code recognized by sensors installed in the learning chambers. After the transponder procedure, subjects were moved from the housing facilities to the experimental rooms. The animals were then transferred to three IntelliCage systems, each housing 10-12 mice randomly drawn from the stocks of their parental lines.

Mice housed in each of the IntelliCages were maintained in a 12:12 light schedule and subjected to a 15 day protocol divided into four phases: simple adaptation, nosepoke adaptation, and place preference learning and reward-motivated discrimination learning (Fig. 2A). During simple adaptation phase (days 1-4), all doors in the learning chambers remained open and access to water was unrestricted. During the nosepoke adaptation phase (days 5-7), all doors were closed and opened only when an animal pokes its nose (nosepoke response) into one of the two openings placed inside learning chambers. When an animal removed the snout from the opening, the door closed automatically. During the simple adaptation and nosepoke adaptation phase each of 8 bottles contained tap water (days 1-7, Fig. 2A). During the place preference learning phase (days 8-10) access to the drinking bottles was restricted to only one of the IntelliCage learning chambers for each mouse.

The corner with water access was assigned randomly, to no more than 3 mice. Such procedure minimized social modulation of learning (Kiryket al. 2011). During reward-motivated discrimination learning tap water in one bottle in the corner was replaced by $10 \%$ sucrose solution, which is strongly preferred by mice (Days 11-15). Animals had a choice between nosepoking (operant response) to the bottle containing tap water or to the bottle containing a reward (sweetened water) placed in the same conditioning corner. They had to remember location of the reward to perform the correct response. The number of visits, nosepokes and tube licks were recorded automatically by the computer controlled IntelliCage system in 12-h time intervals. All raw data were then analyzed by PyMICE - Python library for mice behavioral data analysis (Kowalski et al. 2016).

\section{Morphometrics}

Following measurements of cognitive abilities, animals were killed by cervical dislocation and dissected. Brain, heart, liver and kidneys were excised, blotted from excess fluids and weighed to an accuracy of $0.001 \mathrm{~g}$. 


\section{LTP measurements}

Naive animals were anaesthetized with isoflurane and decapitated. The brains were instantly removed and placed in cold artificial cerebrospinal fluid ACSF ( NaCl $117 \mathrm{mM}, \mathrm{MgSO}_{4} 1.2 \mathrm{mM}, \mathrm{KCl} 4.7 \mathrm{mM}, \mathrm{CaCl}_{2} 2.5$ mM, $\mathrm{NaHCO}_{3} 25 \mathrm{mM}, \mathrm{NaH}_{2} \mathrm{PO}_{4} 1.2 \mathrm{mM}, 10 \mathrm{mM}$ glucose, bubbled with carbogen) and both hemispheres were cut into $400 \mu \mathrm{m}$ coronal slices with a vibratome (LeicaVT1000S). Slices containing hippocampus were placed in a recording interface chamber (Harvard Apparatus) to recover for at least $1.5 \mathrm{~h}$ before the start of recordings. The slices were continuously perfused with carbogenated CSF at $33^{\circ} \mathrm{C}$. Field excitatory postsynaptic potentials (fEPSPs) were recorded using a glass pipette filled with $20 \mathrm{mMNaCl}$ (impendence 1.0-3.0 M $\Omega$ ) from the stratum radiatum in CA1 area of the hippocampus (Fig. 2). To evoke fEPSP, Schafer collateral-commissural afferents were stimulated every $30 \mathrm{~s}$ (test pulses at $0.033 \mathrm{~Hz}, 0.1 \mathrm{~ms}$ ) with bipolar metal electrodes (FHC, USA). The intensity of test stimuli were adjusted to obtain fEPSP with slopes of one-third of the maximal response. After at least $15 \mathrm{~min}$. of stable baseline, LTP was induced tetanically (three trains of $100 \mathrm{~Hz}, 1 \mathrm{~s}$ stimulation, separated by $3 \mathrm{~min}$ ). After the end of the tetanic stimulation, a test pulse was subsequently applied for at least 90 min. Recordings were amplified (EX4-400 Dagan Corporation, USA), digitized (POWER1401, CED, UK) and slopes of fEPSP analyzed on-line and off-line. For analysis of LTP, the response slopes were expressed as a percentage of the average response slopes during the baseline period prior to LTP induction.

\section{Statistical analyses}

Data on BMR and masses of internal organs were analysed by means of ANCOVA with line type affiliation as a fixed factor and body mass as a covariate. In this analysis and the behavioural analyses described below, replicated lines were nested within line types as the random factor of the model (4 replications in the RB and PMR line types, respectively, but 1 line for H-BMR and L-BMR line types, respectively, as they were not replicated; 10 lines in total). The respective error mean square for 10 lines was used as denominator of the $\mathrm{F}$ statistics testing the effect of line affiliation. Hence, the $d f$ for the between line type comparisons was 3 (for the $\mathrm{F}$ numerator) and 6 (for denominator). Likewise, the $d f$ for pairwise t-test comparisons between the line types was 6 .

Repeated measures analysis of covariance (ANCOVA) was used to analyse the between-line type differences in total numbers of visits to all four corners summed over four continuous $12 \mathrm{~h}$ periods of observation, covering the last $24 \mathrm{~h}$ of place preference learning, and first $24 \mathrm{~h}$ of reward-motivated discrimination learning.

For the same period of time we analysed the number of visits to the bottles located in a corner assigned to a given animal. During first two $12 \mathrm{~h}$ periods both bottles in the corner contained water, and subsequently, for the next two $12 \mathrm{~h}$ periods of reward-motivated discrimination learning, one bottle was filled with $10 \%$ sucrose solution. The numbers of correct responses (i.e. nosepokes to the bottle with sucrose) were corrected for (1) the dark and the light phase of the experimental period (nested within the effect of time coded as a fixed factor) (2) numbers of nosepokes to the bottle with tap water located in the same corner coded as a covariate. Initially, we also controlled for possible differences between batches of animals (coded as a random variable) maintained together in the IntelliCage system. However, since this effect was never significant at $\mathrm{p}$ $=0.05$, we dropped it from final analyses. We used an analogously structured model to analyse the number of licks on the bottles containing tap or sweetened water.

Data on LTP were analysed by means of repeated measures ANOVA with line type affiliation as a main factor. In this analysis we compared the LTP slopes between the H-BMR line types along with one, randomly drown RB line as the outgroup. All statistical analyses were carried out by means a mixed model extension of a general linear model (SAS/STATR 14.1 User's Guide).

\section{Result and Discussion}

Mice of the used line types did not differ with respect to body mass. Yet, high BMR (H-BMR) mice were characterized by conspicuously higher BMR then mice of all other line types (Table 1, Fig. 1A). Their metabolically expensive internal organs (liver, heart and kidneys) were also larger than in mice of other line 
types (Fig. 1B-D). Yet, their brains were not significantly larger (Fig. 1E). Thus we did not observe the brain-gut trade-off as predicted by the ET hypothesis.

To compare learning abilities of the line types we trained mice in IntelliCages, an automated system that allows for individual assessment of activity and learning of group-housed mice (Knapska et al.2013). In an initial acclimatization period, mice were able to access water in any of the four corners of the IntelliCage - each corner had two separate bottles with tap water that the mouse could choose between. During the place preference learning, water access for each mouse was restricted to one of the four corners. Next, in the reward-motivated discrimination learning, one of the bottles was filled with a reward - $10 \%$ sucrose solution (Fig. 2A).

To assess reward-motivated discrimination learning we measured the number of nosepokes that opened access to the bottle with sucrose solution (correct responses). In comparison to the previous phase of the training, all mice increased the number of nosepokes to the bottle that now contained the reward. However, high BMR mice accessed the reward more often than their low $\mathrm{BMR}, \mathrm{VO}_{2 \max }$ and randomly bred counterparts (Table 2, Fig. 2B). The results indicate that the high BMR mice learned the rewarded response faster than the other animals. To test whether the improved learning could be attributed to changes in thirst or taste discrimination, the number of licks from the bottles that contained sucrose solution was analysed. We did not observe any differences between the line types in the amount of sweetened water consumed (Fig. 2D). Further, because differences in general activity could potentially influence the obtained results, we compared the numbers of visits to all corners during the reward-motivated discrimination learning phase and the adaptation phase. The rate of visiting corners did not differ between the line types (Table 2), excluding the possibility that the differences in learning could be explained by changes in general activity.

To gain insight into the potential neuronal mechanism underlying observed differences in learning we used long-term potentiation (LTP), a classical model for investigation of activity-dependent synaptic plasticity. We compared effects of repeated high-frequency stimulation of Schaeffer collaterals that make excitatory synapses onto pyramidal cells in the CA1 region of the hippocampus, the brain structure crucial for spatial memory formation. We compared the slope of LTP in the H-BMR mice, L-BMR mice and the animals from one of the randomly bred (non-selected) lines as the outgroup. In line with the behavioural results, the H-BMR mice manifested significantly increased neuronal plasticity $\left(\mathrm{F}_{2,24}=18.4 \mathrm{p}<0.001\right.$, Fig. $\left.2 \mathrm{C}\right)$.

It is important to note that throughout our experiment mice were fed the same diet, so the partial tenet of the ET hypothesis - compensation of the reduced gut by increased food quality could not be tested. Yet, at least in non-mammalian animal models BGTO is likely to occur even without a shift in quality of consumed food, as demonstrated by Kotrschal et al . (2013) Also, as we demonstrated elsewhere (Ksiażek et al. 2009) high BMR mice possess a considerable digestive safety margins, which would have left them an ample potential for gut size reduction envisaged by the brain-gut trade-off.

The costs of increased brain size and CA can be satisfied by (i) reallocation of resources towards brain growth and maintenance from other sinks (other energetically expensive organs, as proposed by the ET hypothesis (Aiello \& Wheeler, 1995) or physiological traits such as immunocompetence (Kotrschal et al. 2016); or by (ii) increasing total energy intake, which may allow to cover the costs of cognitive abilities without the need for reduction of other structures and functions, including digestive abilities. Overall an increase of energy intake is the hallmark of the evolution of endothermy (Polymeropouloset al. 2018), particularly linked with the need to fuel reproduction (Koteja 2000). The high BMR mice are characterized by both increased energy intake and reproductive allocation (Chrzaścik et al. 2014) and increased mass of the gut (Table 1). This points to (i) and suggests that the selection for enhanced CA does not need to involve brain-gut trade-off as an initial step toward the evolution of enhanced CA. The more likely evolutionary scenario would involve initial selection for increased overall energy intake, which would necessitate an increased gut size and BMR (Healy \& Rowe 2007). This selection may have involved an initial increase in neuronal efficiency, if more efficient neurons were metabolically cheaper than an increase of the number of neurons and/or in their size (Herculano-Houzel 2011). Such smarter, but not necessarily bigger brains allowed for foraging on better quality food. Subsequently other trade-offs (such as gut reduction) may have occurred in some lineages, 
such as proto-human apes, allowing for brain size increases.

Acknowledgments

We acknowledge valuable comments by Leszek Kaczmarek and Jan Kozłowski and experimental assistance provided by A. Gebczyński, B. Lewończuk, M. Lewoc, S. Płonowski, and J. Sadowska. Miłka Piszczek greatly helped to edit the paper. Funding: This work was supported by National Research Center NCN 2015/17/B/NZ8/02484 grant. Authors contributions: A.G., E.K. and M.K. conceived of the idea and designed the experiments. All authors participated in the experiments and data analysis. A.G., E.K. and M.K. prepared the manuscript. Competing interests: The authors declare no competing financial interests.Data and materials availability: All raw data are available from Dryad repository (doi:10.5061/dryad.bk3j9kd78).

Tables

Table 1.

ANCOVA results for BMR and anatomical traits

\begin{tabular}{lll}
\hline & Line type & Body Mass \\
\hline Body Mass & $\mathrm{F}_{3,6}=1.69 \mathrm{p}=0.27$ & - \\
BMR & $\mathrm{F}_{3,6}=36.63 \mathbf{p}<\mathbf{0 . 0 0 1}$ & $\mathrm{F}_{1,208}=72.12 \mathrm{p}<0.001$ \\
Brain & $\mathrm{F}_{3,6}=1.03 \mathrm{p}=0.44$ & $\mathrm{~F}_{1,135}=5.96 \mathrm{p}=0.016$ \\
Liver & $\mathrm{F}_{3,6}=10.18 \mathbf{p}=\mathbf{0 . 0 0 9}$ & $\mathrm{F}_{1,132}=28.56 \mathrm{p}<0.001$ \\
Heart & $\mathrm{F}_{3,6}=26.54 \mathbf{p}<\mathbf{0 . 0 0 1}$ & $\mathrm{F}_{1,137}=13.72 \mathrm{p}<0.001$ \\
Kidneys & $\mathrm{F}_{3,6}=6.34 \mathbf{p}=\mathbf{0 . 0 3}$ & $\mathrm{F}_{1,135}=48.85 \mathrm{p}<0.001$ \\
\hline
\end{tabular}

\section{Table 2.}

Repeated measures ANCOVA results for behavioural tests

\begin{tabular}{lllll}
\hline & Line type & Time(Phase) & Time & Time $\times$ Line Type \\
\hline Activity & $\mathrm{F}_{3,6}=2.76 \mathrm{p}=0.13$ & $\mathrm{~F}_{2,845}=511.98 \mathrm{p}<$ & $\mathrm{F}_{1,845}=19.1 \mathrm{p}<$ & $\mathrm{F}_{3,845}=4.41 \mathrm{p}=$ \\
& & 0.001 & 0.001 & 0.004 \\
Correct & $\mathrm{F}_{3,6}=6.91 \mathrm{p}=$ & $\mathrm{F}_{2,764}=118.0 \mathrm{p}$ & $\mathrm{F}_{1,764}=407.45 \mathrm{p}$ & $\mathrm{F}_{3,764}=24.01 \mathrm{p}$ \\
Nosepokes $^{\mathrm{a}}$ & $\mathbf{0 . 0 2 3}$ & $<0.001$ & $<0.001$ & $<0.001$ \\
Licks $^{\mathrm{b}}$ & $\mathrm{F}_{3,6}=0.30 \mathrm{p}=0$. & $\mathrm{F}_{2,758}=225.34 \mathrm{p}<$ & $\mathrm{F}_{1,758}=212.06 \mathrm{p}<$ & $\mathrm{F}_{3,758}=5.63 \mathrm{p}<$ \\
& 82 & 0.001 & 0.001 & 0.001 \\
\hline
\end{tabular}

a the numbers of correct responses (i.e. nosepokes to the bottle with sucrose) were corrected for numbers of nosepokes to the bottle with tap water located in the same corner (used as a covariate, significant at $\mathrm{p}<0.001)$.

b the numbers of licks the bottle with sucrose were corrected for numbers of licks to the bottle with tap water located in the same corner (used as a covariate, significant at $\mathrm{p}<0.001$ ).

Figures

Fig. 1. (A) Basal metabolic rate (BMR) in line types of mice selected for high (H-BMR) or low (L-BMR) Basal Metabolic Rate (BMR), Peak Metabolic Rate (PMR aka VO $\mathrm{V}_{2 \mathrm{max}}$ ) and random-bred (RB) lines; (B-D) their masses of internal organs and (E) brain mass. Values are body mass adjusted means with standard errors calculated from ANCOVA. Figure bars labelled with different letters differ significantly from each 
other at $\mathrm{p}=0.05$.
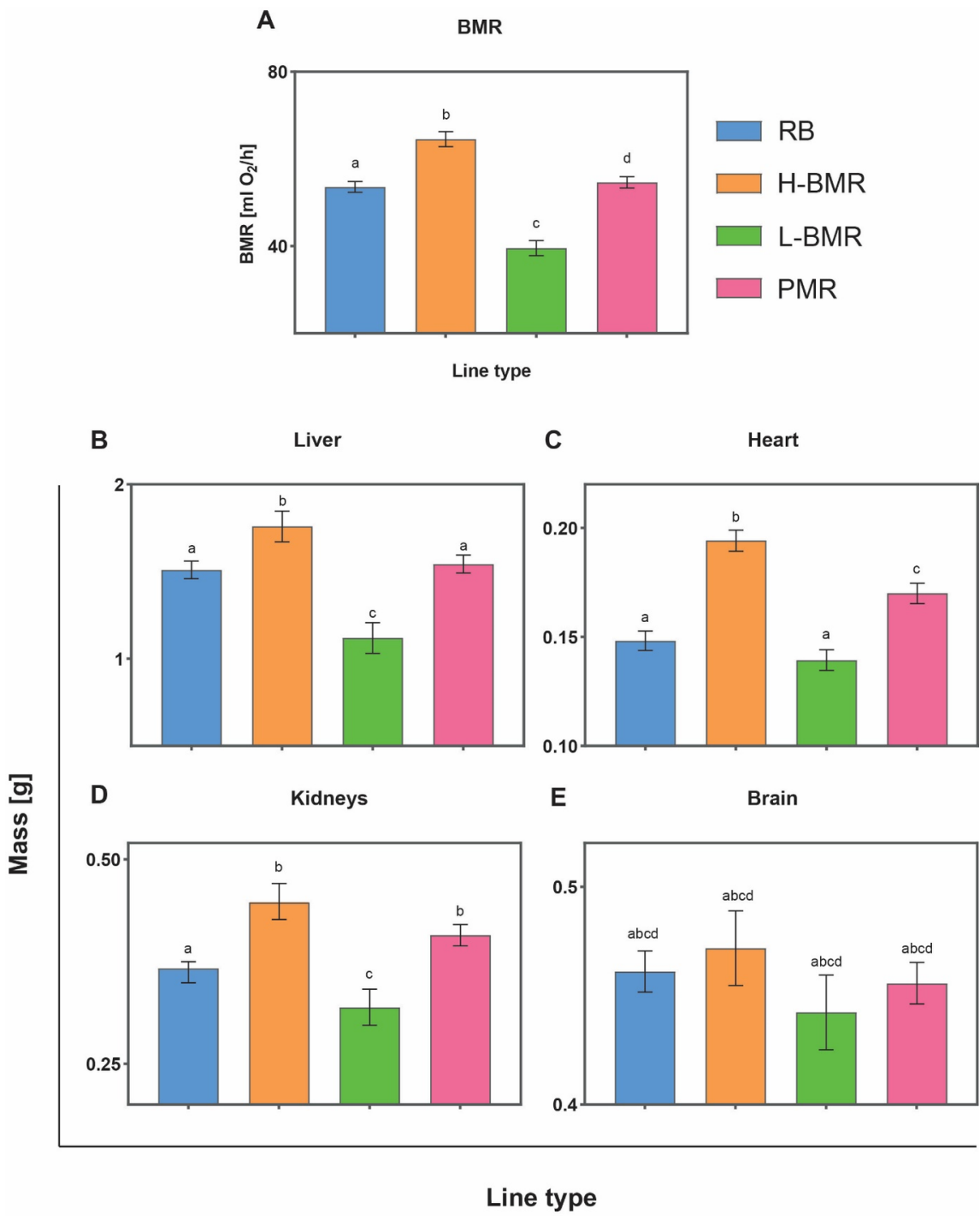

Fig. 2. (A) Scheme of the experiments in the IntelliCage. Mice were subjected to experimental procedures that consisted of adaptation and learning phases. After adaptation phases (simple adaptation phase days 1-4 and nosepoke adaptation phase days 5-7, depicted as days 1-7) mice were subject to place preference learning (days 8-10) and reward-motivated discrimination learning (days 11-15). Results of place discrimination learning: (B) Number of nosepoke responses giving access to the bottle that contained tap water in preference learning, then sweetened water (10\% sucrose) in reward-motivated discrimination learning (aligned by vertical dashed line with the timeline of experiment). The number of correct responses was corrected for the number of nosepokes to the bottle with tap water placed in the same corner. Values labelled with different letters differed from each other at $\mathrm{p}=0.05$ (pairwise a-priori t-test with $\mathrm{df}=6$ ). (D) Number of licks from the bottle that contained first tap then sweetened water in adaptation and training, respectively. (C) LTP recorded at the Shaffer correlates in the hippocampus. The time course of maximal EPSP slopes was normalized to baseline in the CA1 region of the hippocampus. Long-term potentiation was induced by high-frequency stimulation (HFS; $3 \times 100 \mathrm{~Hz}$ ) of the Schaffer collaterals in slices from the H-BMR (orange squares, $\mathrm{n}=10$ ), L-BMR (green triangle, $\mathrm{n}=8$ ) and the RB mouse line (blue circles, $\mathrm{n}=7$ ). The slopes labeled with different 
letters differed from each other at $\mathrm{p}=0.05$ by the Tukey post-hoc test. (E) Representative traces of fEPSP $10 \mathrm{~min}$ before (black) and 15 and $90 \mathrm{~min}$ after (grey) the induction of LTP are shown. Scale bars $=2 \mathrm{mV}$ and $5 \mathrm{~ms}$.

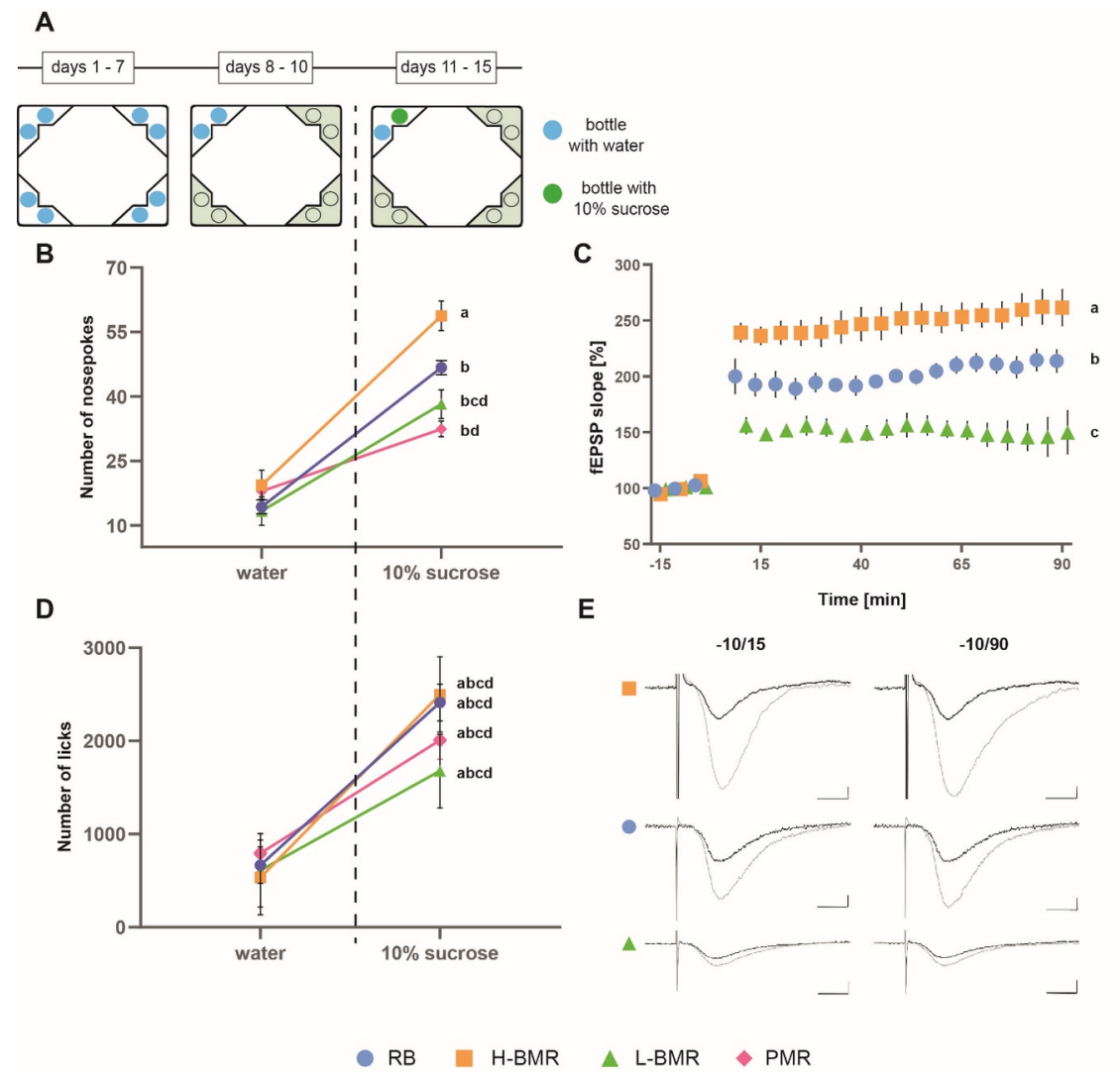

\section{References}

1. Aiello, L.C. \& Wheeler, P. (1995) The Expensive-Tissue Hypothesis: The brain and the digestive system in human and primate evolution. Curr. Anthropol. 36, 199-221.

2. Bennett, A. F., \& Ruben, J. A. (1979). Endothermy and activity in vertebrates. Science 206 , 649-653.

3. Chrzaścik, K.M., Sadowska, E.T., Rudolf, A. \& Koteja, P. (2014) Learning ability in bank voles selected for high aerobic metabolism, predatory behaviour and herbivorous capability. Physiol. Behav.135, $143-151$.

4. Galsworthy, M.J., Amrein, I., Kuptsov, P.A., Poletaeva, I.I., Zinn, P., Rau, A., Vyssotski, A., \& Lipp H.P. (2005) A comparison of wild-caught wood mice and bank voles in the Intellicage: assessing exploration, daily activity patterns and place learning paradigms.Behav. Brain Res. 157, 211-217.

5. Garland T, \& Rose MR Ed., Experimental Evolution: Concepts, Methods, and Applications of Selection Experiments . (University of California Press, 2009).

6. Gebczyński, A.K. \& Konarzewski, M. (2009) Locomotor activity of mice divergently selected for basal metabolic rate: a test of hypotheses on the evolution of endothermy. J. Evol. Biol. 22, 1212-1220.

7. Healy SD, \& Rowe CA (2007) A critique of comparative studies of brain size, Proc. R. Soc. Lond. B Biol. Sci. , 274(1609): 453-464.

8. Herculano-Houzel S (2011) Scaling of brain metabolism with a fixed energy budget per neuron: Implications for neuronal activity, plasticity and evolution. PLoS ONE 6 e17514.

9. Isler K, \& van Schaik C.P (2006) Metabolic costs of brain size evolution. Biol. Lett. 2(4): 557-560.

10. Kiryk, A., Mochol, G., Filipkowski, R.K., Wawrzyniak, M., Lioudyno, V., Knapska, E., Gorkiewicz, T., 
Balcerzyk, M., Leski, S., Van Leuven, F., Lipp, H. P., Wojcik, \& D.K., Kaczmarek, L. (2011) Cognitive abilities of Alzheimer's disease transgenic mice are modulated by social context and circadian rhythm. Curr. Alzheimer Res . 8, 883-892.

11. Knapska, E., Walasek, G., Nikolaev, G,E., Neuhäusser-Wespy, F., Lipp, H.P., Kaczmarek, L., \& Werka, T. (2006) Differential involvement of the central amygdala in appetitive versus aversive learning," Learn. Mem. Cold Spring Harb. N 13, 192-200.

12. Knapska, E., Lioudyno, V., Kiryk, A., Mikosz, M., Górkiewicz, T., Michaluk, P., Gawlak, M., Chaturvedi, M., Mochol, G., Balcerzyk, M., Wojcik, D.K., Wilczynski, G.M. \& Kaczmarek, L. (2013) Reward learning requires activity of matrix metalloproteinase-9 in the central amygdala, Neurosci. Off. J. Soc. Neurosci. 33, 14591-14600.

13. Konarzewski, M. \& Diamond, J. (1995) Evolution of basal metabolic rate and organ masses in laboratory mice. Evolution 49, 1239-1248.

14. Koteja, P. (2000) Energy assimilation, parental care and the evolution of endothermy. Proc. R. Soc. Lond. B Biol. Sci. 267, 479-484 (2000).

15. Koteja, P. (2004) The evolution of concepts on the evolution of endothermy in birds and mammals," Physiol. Biochem. Zool.77, 1043-1050.

16. Kotrschal, A., Rogell, B., Bundsen, A., Svensson, B., Zajitschek, S., Brännström, I., Immler, S., Maklakov, A.A., \& Kolm, N. (2013) Artificial selection on relative brain size in the guppy reveals costs and benefits of evolving a larger brain. Curr. Biol. 23, 168-171.

17. Kotrschal, A., Kolm, N., \& Penn, D.J. (2016) Selection for brain size impairs innate, but not adaptive immune responses. Proc. R. Soc. B Biol. Sci. 283, 20152857.

18. Kowalski, J., Łeski, S., Puścian, A. (2016) PyMICE: 0.2.3 .

19. Ksiażek, A., Czarniecki, J., \& Konarzewski, M. (2009) Phenotypic flexibility of traits related to energy acquisition in mice divergently selected for basal metabolic rate (BMR). J. Exp. Biol. 212, 808-814.

20. Ksiażek, A., Konarzewski, M., \& Lapo, I.B. (2004) Anatomic and energetic correlates of divergent selection for basal metabolic rate in laboratory mice. Physiol. Biochem. Zool. Ecol. Evol. Approaches 77, 890-899.

21. Lovegrove, B., G. A phenology of the evolution of endothermy in birds and mammals. Biol. Rev. 92 , 1213-1240 (2017).

22. Navarrete, A., van Schaik, C.P., \& Isler, K. (2011) Energetics and the evolution of human brain size. Nature 480, 91-93.

23. Polymeropoulos, E. T., Oelkrug, R., \& Jastroch, M. (2018) Editorial: The Evolution of EndothermyFrom Patterns to Mechanisms Front. Physiol. 9.

24. Pontzer, H., Brown, M.H., Raichlen, D.A., Dunsworth, H., Hare, B., Walker, K., Luke, A., Dugas, L.R., Durazo-Arvizu, R., Schoeller, D., Plange-Rhule, J., Bovet, P., Forrester, T.E., Lambert, E.V., Thompson, M.E., Shumaker, R.W., Ross, \& S.R. (2016) Metabolic acceleration and the evolution of human brain size and life history. Nature 533, 390-392.

25. Rose, M.R., Mueller, L.D., \& Stearns, S.C. (1993) The evolution of life histories. (Oxford University Press, London 1992). J. Evolut. Biol . 6, 304-306.

26. Sadowska, J., Gebczyński, A.K., \& Konarzewski, M. (2017) Selection for high aerobic capacity has no protective effect against obesity in laboratory mice. Physiol. Behav. 175, 130-136.

27. SAS/STAT\& 14.1 User's Guide. The MIXED Procedure 2015. SAS Institute Inc., Cary, NC, USA.

28. Sayol, F., Downing, P.A., Iwaniuk, A. N., Maspons, J. \& Sol, D. (2018). Predictable evolution towards larger brains in birds colonizing oceanic islands. Nature Comm . 9, 2820.

29. Withers, P.C. (1997) Measurement of VO2, VCO2, and evaporative water loss with a flow through mask. J. Appl. Physiol. 42, 120-123. 